\title{
The Inverse Dynamics of a 3-DOF Parallel Mechanism Based on Analytical Forward Kinematics
}

\author{
Ke Wang \\ Changzhou University \\ Ju Li \\ Changzhou University \\ Huiping Shen ( $\nabla$ shp65@126.com ) \\ Changzhou Uniersity https://orcid.org/0000-0002-1563-0924 \\ Jingjing You \\ Nanjing Forestry University \\ Ting-Li Yang \\ Changzhou University
}

Original Article

Keywords: parallel mechanism, dynamic model, Newton-Euler method, simulation verification

Posted Date: August 13th, 2020

DOI: https://doi.org/10.21203/rs.3.rs-57314/v1

License: (c) (1) This work is licensed under a Creative Commons Attribution 4.0 International License. Read Full License 


\section{The Inverse Dynamics of a 3-DOF Parallel Mechanism Based on Analytical Forward Kinematics}

Ke Wang, born in 1994, is currently a engineer at Black \& Decker(Suzhou) Technology Limited Company, China. He received his master degree from Changzhou University, China, in 2020.

E-mail: wangk1994@163.com

Ju Li, born in 1981, is currently a Vice Professor at Changzhou University, China. Her main research interests include parallel mechanism, kinematics, E-mail: wangju0209@163.com

Huiping Shen, born in 1965, is currently a Professor and a PhD candidate supervisor at Changzhou University, China. His main research interests include parallel mechanism, kinematics, topology.

E-mail: $\underline{\text { shp65@126.com }}$

Jingjing You, born in 1985, is currently a Vice Professor and a Master supervisor at College of Mechanical and Electronic Engineering, Nanjing Forestry University, China. E-mail: youjingjing251010@126.com

Ting-Li Yang, born in 1940, is currently a Guest Professor. His main research interest include modern mechanisms, basic theory of robotic mechanisms. E-mail:yangti@126.com

Corresponding author: Huiping Shen E-mail: shp65@126.com 


\title{
The Inverse Dynamics of a 3-DOF Parallel Mechanism Based on Analytical Forward Kinematics
}

\author{
Ke Wang ${ }^{1} \bullet J_{u} \mathrm{Li}^{1} \bullet$ Huiping Shen ${ }^{1} \bullet$ Jingjing You ${ }^{2} \cdot$ Ting-li Yang${ }^{1}$
}

Received on xx xx, 2020; accepted on xx xx, 2020

\begin{abstract}
A new type of 3-dof parallel mechanism(PM) with analytical forward displacement analysis is proposed. The reverse dynamic equation of the PM is solved. Different from the traditional dynamic analysis using inverse kinematics, the displacement, velocity and acceleration equations of the PM are established and solved by forward kinematics. The inverse dynamic equation of the PM is constructed and solved by analyzing the forces on each link and based on Newton-Euler method. The correctness of the dynamic model is verified by an example using MATLAB and ADAMS. The maximum driving force error of each actuated pair is $1.32 \%, 5.8 \%$ and $5.2 \%$ respectively.This paper provides a theoretical basis for the design, manufacture and application of the PM.
\end{abstract}

Keywords: parallel mechanism • dynamic model $\bullet$ Newton-Euler method $\bullet$ simulation verification

\section{Introduction ${ }^{1}$}

Compared with the series mechanism, the parallel mechanism (PM) has the advantages of compact structure, high rigidity and motion accuracy, etc., The topic has attracted extensive attention from the academic and industrial community for many years [1-3]. At present, research on PMs mainly focuses on the topology, kinematics, dynamics and control[4-6].The dynamic analysis mainly studies the relationship between the input forces and the output forces. This is the determination of the maximum load carrying capacity and reasonable design of the driver during the development of parallel robots. According to this, the constraint reaction force of the motion pair solved during the analysis process plays an important role in the design, mechanical efficiency estimation, friction calculation, and

\footnotetext{
Huiping Shen

Shp65@126.com

School of Mechanical Engineering, Changzhou University, Changzhou 213164, China

2 College of Mechanical and Electronic Engineering, Nanjing Forestry University, Nanjing 210037, China
}

mechanical vibration research of the various parts of the PM. Therefore, for a PM to be developed, it is necessary to establish a dynamic model that can accurately and meet the requirements of real-time control.

In terms of modeling methods, the commonly used dynamic modeling methods are Lagrangian method, universal equations of dynamics, Newton-Euler method, etc.[7-9]. Among them, the universal equations of dynamics and Lagrange method are based on the system's virtual displacement and kinetic and potential energy respectively to build a simple dynamic model. While the Newton-Euler method can obtain the force of each joint by analyzing each member separately, and then establishes a complete dynamic model by eliminating the interaction force of each member [10], it can also solve the support force and moment of force between members.

In terms of research objects, most of the kinetic analysis mainly focus on the 6-DOF Stewart PM [11-13]. For 5-DOF PM, Chen et.al [14] use the universal equations of dynamics to analyze the 4-UPS-UPU PM. Li et.al[10] used the Newton-Euler method to analyze the dynamics of the 5-PSS / UPU PM. For 4-DOF PM, Geng[15] used Newton-Euler to analyze the 4UPS-UPR PM. In terms of 3-DOF, Li et.al[16] used the Newton-Euler method to analyze the dynamics of a 3-RPS PM. Liu et.al [17] performed a Lagrangian method to analyze the dynamics of a 3-RRS PM.

In this paper, a 3-DOF 2T1R PM[18] with a forward analytical position solution is proposed. Firstly, the kinematics of the PM is analyzed.Then, force analysis is carried out for each component, and the dynamic model is established by Newton-Euler method.Finally, the correctness of the modeling method is verified by comparing the results of Matlab calculation and ADAMS simulation.It provides the theoretical basis of mechanics for the design, manufacture and application of the PM. 


\section{Mechanism architecture}

The PM shown in Figure 1 consists of a moving platform, a base, and a complex branch chain and an unconstrained branch PSS (Prismatic pair - Spherical joint - Spherical joint) connecting the moving platform and the base. The sub-PM and an revolute pair are connected in series, where the two branches of the sub-PM are $\mathrm{SOC}_{1}\left\{-\mathrm{P} \perp \mathrm{P}^{(4 \mathrm{R})}-\right\}, \mathrm{SOC}_{2}\{-\mathrm{P} \perp \mathrm{R}$ $\| \mathrm{R}\}$, where $\mathrm{P}^{(4 \mathrm{R})}$ indicates that a parallelogram composed of 4 revolute pairs that is equivalent to a prismatic pair $\mathrm{P}$, while SOC means single-open-chain that consists of link and pair in serial way. This PM can generate two translations in the $Y$ and $Z$ directions and a rotation output about the $Y$ axis. The PM is referred to as the $2 \mathrm{~T} 1 \mathrm{R}$ PM ${ }^{[18]}$.

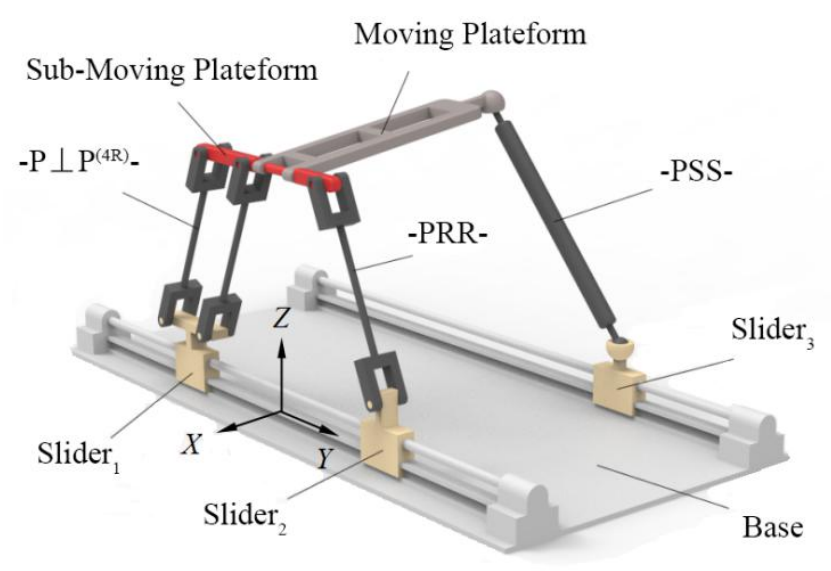

Figure $13 \mathrm{D}$ model of a 2T1R PM

\subsection{Kinematics analysis}

The coordinate system shown in Figure 2 is established. The base coordinate system $O-X Y Z$ is established with the origin at the center point $\mathrm{O}$ of the guide rail where the driving pair $A_{1} 、 A_{2}$ is located, the positive half of the Y-axis is from $O$ to $A_{2}$, and the positive half of the $Z$-axis is vertical upward.The moving coordinate system $o-x y z$ is established with the origin at $o$ point that is the center point $\mathrm{O}$ of the line $\mathrm{C}_{12} \mathrm{C}_{2}$ on the sub-moving platform. The positive half of the $y$-axis is from the origin $o$ to $C_{2}$, and the $\mathrm{z}$-axis is perpendicular to the plane of the moving platform. While the $X$ and $x$ axis direction meet the right-hand screw rule, the geometry parameters of each component are shown in Figure 2.

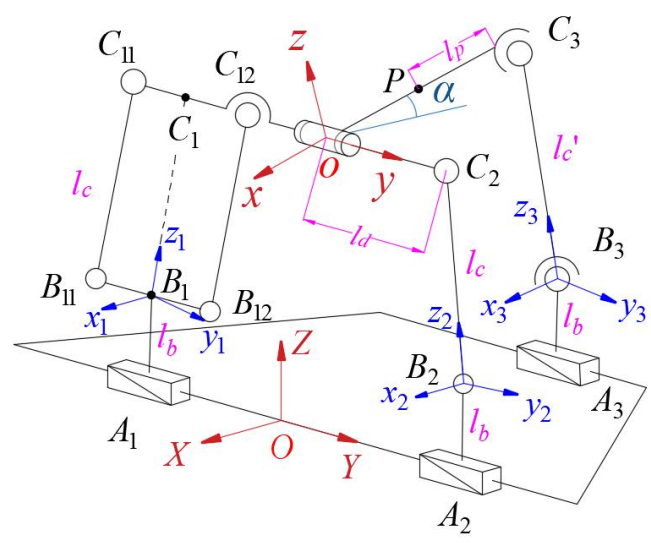

Figure 2 Schematic diagram of 2T1R PM

Establish the local coordinate system of each link $B_{i} C_{i}$ $(i=1,2,3)$. The $x_{1}$ axis of the coordinate system $B_{1}-x_{1} y_{1} z_{1}$ is parallel to the $X$ axis of the base coordinate system, and the positive half of the $z_{1}$ axis is pointed from $B_{1}$ to $C_{1}$; The $\mathrm{x}_{2}$ axis of the coordinate system $B_{2}-x_{2} y_{2} z_{2}$ is parallel to the $X$ axis of the base coordinate system, and the positive half of the $z_{2}$ axis is pointed from $B_{2}$ to $C_{2}$, where the $y$ axis of each coordinate system meets the right-hand screw rule; The positive half of the $z_{3}$ axis of the coordinate system $B_{3}-x_{3} y_{3} z_{3}$ is pointed from $B_{3}$ to $C_{3}$, and the $x_{3}$ axis lies in the $X O Z$ plane and the angle with the $X$ axis is $\theta_{1}$, its Euler transformation relative to the base coordinate system is shown in Figure 3, from which the coordinate transformation matrix from the coordinate system $\left\{B_{3}\right\}$ to the base coordinate system $O-X Y Z$ is:

$$
{ }^{O} \boldsymbol{R}_{l_{3}}=\boldsymbol{R}\left(Y, \theta_{1}\right) \boldsymbol{R}\left(x_{3}^{\prime}, \theta_{2}\right)
$$

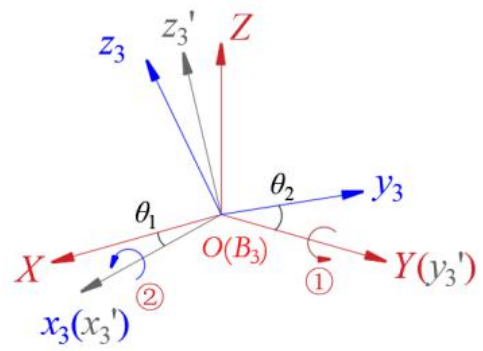

Figure 3 Coordinate system $B_{3}-x_{3} y_{3} z_{3}$ Euler transform

\subsection{Forward Position Solution}

The forward kinematics of PM is to solve the position and orientation of the moving platform when the structural parameters and input of the mechanism are given.

Based on the constrained length of the bars, we can get: 
$f_{1}: l_{c}^{2}-\left(z-l_{b}\right)^{2}-\left(y-l_{d}-l_{1}\right)^{2}=0$

$f_{2}: l_{c}^{2}-\left(z-l_{b}\right)^{2}-\left(l_{2}-y-l_{d}\right)^{2}=0$

$f_{3}:\left(-2 l_{p} \cos \alpha-x_{A_{3}}\right)^{2}+\left(y-l_{3}\right)^{2}+\left(z+2 l_{p} \sin \alpha-l_{b}\right)^{2}=l_{c}^{2}$

From Eq. (1), the coordinates of point $o$ are:

$$
\begin{aligned}
& y=\frac{l_{1}+l_{2}}{2} \\
& z=\sqrt{l_{c}{ }^{2}-\left(\frac{l_{2}-l_{1}}{2}-l_{d}\right)^{2}}+l_{b}
\end{aligned}
$$

Angle $\alpha$ of moving platform is:

$$
\alpha=2 \arctan \left(\frac{-B \pm \sqrt{B^{2}-C^{2}+A^{2}}}{C-A}\right)
$$

where,

$$
\begin{aligned}
& A=4\left(z-l_{b}\right) \cdot l_{p} \\
& B=4 x_{A_{3}} \cdot l_{p} \\
& C=-4 l_{p}{ }^{2}-x_{A_{3}}^{2}-\left(y-l_{3}\right)^{2}-\left(z-l_{b}\right)^{2}+l_{c}{ }^{\prime 2}
\end{aligned}
$$

\subsection{Velocity and acceleration analysis}

\subsubsection{Velocity and acceleration of the moving platform}

Taking the time derivative of Eq. (2) (3), the output velocity and acceleration of the moving platform can be obtained as

$$
\begin{aligned}
& \chi=\left[\begin{array}{lll}
\dot{y} & \dot{z} & \dot{\alpha}
\end{array}\right]^{T} \\
& \dot{\chi}=\left[\begin{array}{lll}
\ddot{y} & \ddot{z} & \ddot{\alpha}
\end{array}\right]^{T}
\end{aligned}
$$

\subsubsection{Velocity and acceleration of members}

(1) Velocity and acceleration of member $B_{1} C_{1}$

Because the movements of the $B_{11} C_{11}$ and $B_{12} C_{12}$ rods are the same, the two rods are equivalent to the $\operatorname{rod} B_{1} C_{1}$ for analysis.

The velocity of the point $C_{1}$ is:

$$
v_{c_{1}}=v_{o}=v_{1}+\omega_{l_{1}} \times c_{1} \cdot l_{c}
$$

Where, $\boldsymbol{v}_{o}$ is the linear velocity of point $o ; \boldsymbol{v}_{1}$ is the linear velocity of the driving pair $A_{1} ; c_{1}$ and $\omega_{l_{1}}$ are respectively the linear and angular velocity of the $\operatorname{rod} B_{1} C_{1}$.

The angular velocity of the rod $B_{1} C_{1}$ can be determined by taking the cross product of the two sides of Eq.(4) with $c_{1}$,which yields:

$$
\omega_{l_{1}}=\frac{c_{1} \times\left(v_{c_{1}}-v_{1}\right)}{l_{c}}
$$

Substituting Eq.(5) into Eq.(6) to obtain the velocity of the center of mass of the $\operatorname{rod} B_{1} C_{1}$,

$$
\boldsymbol{v}_{l_{1}}=\boldsymbol{v}_{1}+\omega_{l_{1}} \times \boldsymbol{c}_{1} \cdot \frac{l_{c}}{2}
$$

Taking the time derivative of Eq. (4), the acceleration of the $C_{1}$ can be obtained as:

$$
\boldsymbol{a}_{c_{1}}=\boldsymbol{a}_{o}=\boldsymbol{a}_{1}+\boldsymbol{\varepsilon}_{l_{1}} \times \boldsymbol{c}_{1} \cdot l_{c}+\boldsymbol{\omega}_{l_{1}} \times\left(\omega_{l_{1}} \times \boldsymbol{c}_{1}\right) \cdot l_{c}
$$

The angular acceleration of the $\operatorname{rod} B_{1} C_{1}$ can be determined by taking the cross product of the two sides of Eq.(7) with $c_{1}$,which yields:

$$
\boldsymbol{\varepsilon}_{l_{1}}=\frac{\boldsymbol{c}_{1} \times\left(\boldsymbol{a}_{o}-\boldsymbol{a}_{1}\right)}{l_{c}}=\frac{\tilde{c}_{1} \cdot\left(\boldsymbol{a}_{o}-\boldsymbol{a}_{1}\right)}{l_{c}}
$$

Where, $\widetilde{c}_{1}$ is the skew symmetric matrix associated with the vector $\boldsymbol{c}_{1}$.

Taking the time derivative of Eq. (4), the centroid acceleration of $\operatorname{rod} B_{1} C_{1}$ can be obtained as:

$$
a_{l_{1}}=a_{1}+\varepsilon_{l_{1}} \times c_{1} \cdot \frac{l_{c}}{2}+\omega_{l_{1}} \times \frac{l_{c}}{2}\left(\omega_{l_{1}} \times c_{1}\right)=\frac{1}{2}\left(a_{1}+a_{o}\right)
$$

(2) Velocity and acceleration of member $B_{2} C_{2}$

Similarly, using the same method as the velocity and acceleration of the $\operatorname{rod} B_{1} C_{1}$, the angular velocity of the rod $B_{2} C_{2}$ can be obtained as:

$$
\omega_{l_{2}}=\frac{\boldsymbol{c}_{2} \times\left(\boldsymbol{v}_{c_{2}}-\boldsymbol{v}_{2}\right)}{l_{c}}
$$

The centroid velocity of the $\operatorname{rod} B_{2} C_{2}$ :

$$
\boldsymbol{v}_{l_{2}}=\boldsymbol{v}_{2}+\omega_{l_{2}} \times \boldsymbol{c}_{2} \cdot \frac{l_{c}}{2}
$$

Angular acceleration of $\operatorname{rod} B_{2} C_{2}$ :

$$
\boldsymbol{\varepsilon}_{l_{2}}=\frac{\boldsymbol{c}_{2} \times\left(\boldsymbol{a}_{o}-\boldsymbol{a}_{2}\right)}{l_{c}}=\frac{\widetilde{\boldsymbol{c}}_{2} \cdot\left(\boldsymbol{a}_{o}-\boldsymbol{a}_{2}\right)}{l_{c}}
$$

Centroid acceleration of $\operatorname{rod} B_{2} C_{2}$ :

$$
\boldsymbol{a}_{l_{2}}=\boldsymbol{a}_{2}+\boldsymbol{\varepsilon}_{l_{2}} \times \boldsymbol{c}_{2} \cdot \frac{l_{c}}{2}+\boldsymbol{\omega}_{l_{2}} \times \frac{l_{c}}{2}\left(\boldsymbol{\omega}_{l_{2}} \times \boldsymbol{c}_{2}\right)=\frac{1}{2}\left(\boldsymbol{a}_{2}+\boldsymbol{a}_{o}\right)
$$

(3) Velocity and acceleration of $\operatorname{rod} B_{3} C_{3}$

The velocity of the point $C_{3}$ is

$$
v_{c_{3}}=v_{o}+\omega_{o} \times c_{p}=v_{3}+\omega_{l_{3}} \times c_{3} \cdot l_{c}^{\prime}
$$


The angular velocity of the $\operatorname{rod} B_{3} C_{3}$ can be determined by taking the cross product of the two sides of Eq.(14) with $c_{3}$,which yields:

$$
\omega_{l_{3}}=\frac{c_{3} \times\left(v_{c_{3}}-v_{3}\right)}{l_{c}{ }^{\prime}}
$$

Substituting Eq.(15) into Eq.(16) gives the velocity at the centroid of $\operatorname{rod} B_{3} C_{3}$.

$$
v_{l_{3}}=v_{3}+\omega_{l_{3}} \times c_{3} \cdot \frac{l_{c}{ }^{\prime}}{2}
$$

Taking the time derivative of Eq. (14), the acceleration of the $C_{3}$ can be obtained as:

$$
\begin{aligned}
& \boldsymbol{a}_{c_{3}}=\boldsymbol{a}_{o}+\boldsymbol{\varepsilon}_{o} \times \boldsymbol{c}_{p}+\omega_{o} \times\left(\omega_{o} \times c_{p}\right) \\
& =\boldsymbol{a}_{3}+\varepsilon_{l_{3}} \times c_{3} \cdot l_{c}{ }^{\prime}+\omega_{l_{3}} \times\left(\omega_{l_{3}} \times c_{3}\right) l_{c}{ }^{\prime}
\end{aligned}
$$

The angular acceleration of the $\operatorname{rod} B_{3} C_{3}$ can be determined by taking the cross product of the two sides of Eq.(17) with $c_{3}$,which yields:

$$
\boldsymbol{\varepsilon}_{l_{3}}=\frac{\boldsymbol{c}_{3} \times\left(\boldsymbol{a}_{c_{3}}-\boldsymbol{a}_{3}\right)}{l_{c}{ }^{\prime}}=\frac{\tilde{c}_{3} \cdot\left(\boldsymbol{a}_{c_{3}}-\boldsymbol{a}_{3}\right)}{l_{c}{ }^{\prime}}
$$

Taking the time derivative of Eq. (16), the acceleration of the centroid of $B_{3} C_{3}$ can be obtained as

$$
\boldsymbol{a}_{l_{3}}=\boldsymbol{a}_{3}+\boldsymbol{\varepsilon}_{l_{3}} \times \boldsymbol{c}_{3} \cdot \frac{l_{c}{ }^{\prime}}{2}+\omega_{l_{3}} \times \frac{l_{c}{ }^{\prime}}{2}\left(\omega_{l_{3}} \times c_{3}\right)=\frac{1}{2}\left(a_{3}+a_{c_{3}}\right)
$$

\section{Dynamics modeling of mechanism}

When using the Newton-Euler method, the friction of each moving pair is not considered, then the Newton-Euler equation of each member is established.Then the dynamic model of the PM is obtained by eliminating the internal forces between the members.Finally, the relationship between the driving force and the external forces of the moving platform is obtained, which is illustrated as follows.

\subsection{Dynamic equation of moving platform}

As shown in Figure 4, the gravity of the moving platform is $\boldsymbol{m g}$, the constraint force of the sub-moving platform is $\boldsymbol{F}_{\boldsymbol{a} 1}$, and the constraint force of the PSS branch chain is $\boldsymbol{F}_{\boldsymbol{a} 2}$. The external force and moment of the moving platform are $\boldsymbol{F}_{w}$ and $\boldsymbol{M}_{w}$ respectively.

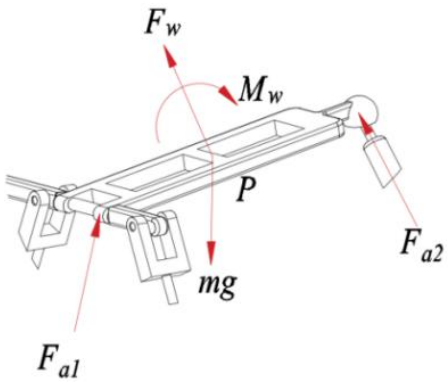

Figure 4 Force analysis of the moving platform

The dynamic equation of the moving platform is:

$$
\begin{gathered}
\boldsymbol{F}_{w}+\boldsymbol{F}_{a 1}+\boldsymbol{F}_{a 2}+m g=m \boldsymbol{a}_{p} \\
\boldsymbol{M}_{w}-\frac{1}{2} \boldsymbol{c}_{p} \times \boldsymbol{F}_{a 1}+\frac{1}{2} \boldsymbol{c}_{p} \times \boldsymbol{F}_{a 2}={ }^{o} \boldsymbol{I}_{p} \boldsymbol{\varepsilon}_{p}+\boldsymbol{\omega}_{p} \times\left({ }^{o} \boldsymbol{I}_{p} \boldsymbol{\omega}_{p}\right)
\end{gathered}
$$

Where, ${ }^{o} \boldsymbol{I}_{p}={ }^{o} \boldsymbol{R}_{p} \boldsymbol{I}_{p}{ }^{o} \boldsymbol{R}_{p}^{T} ;{ }^{o} \boldsymbol{R}_{p}$ is the transformation matrix of the moving coordinate system to the base coordinate system; ${ }^{o} \boldsymbol{I}_{p}$ is the inertia tensor of the moving platform in the base frame; $\boldsymbol{I}_{p}$ is the inertia tensor of the moving platform in the local frame; $c_{p}$ is the position vector from the center of mass of the moving platform to the center of the spherical joint on the moving platform.

\subsection{Dynamic equation of the sub-moving platform}

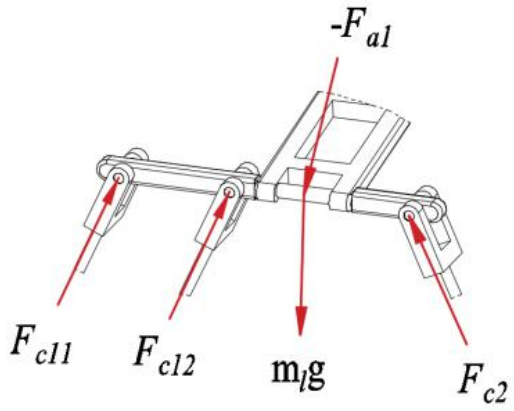

Figure 5 Force analysis of sub-moving platform

As shown in Figure 5, the dynamic equation of the sub-moving platform can be written as follows:

$$
-\boldsymbol{F}_{a 1}+m_{l} \boldsymbol{g}+\boldsymbol{F}_{c 11}+\boldsymbol{F}_{c 12}+\boldsymbol{F}_{c 2}=m_{l} \boldsymbol{a}_{o}
$$

Where $\boldsymbol{F}_{\boldsymbol{c 1 1}}, \boldsymbol{F}_{\boldsymbol{c} 12}, \boldsymbol{F}_{\boldsymbol{c} 2}$ are constraint forces of the active link $B_{i} C_{i}(i=1,2)$ in the sub-moving platform; $m_{l}$ is mass of the 
sub-moving platform; $-\boldsymbol{F}_{a l}$ is the reaction force of the moving platform.

\subsection{Dynamic equation of connecting rod}

The R-R-link is subject to the constraint reaction force of the sub-moving platform $-\boldsymbol{F}_{c i}(i=1,2)$, its own gravity $m_{c} g$, and the constraint force $\boldsymbol{F}_{b i}(\mathrm{i}=1,2)$, and its force analysis is shown in Figure 6.

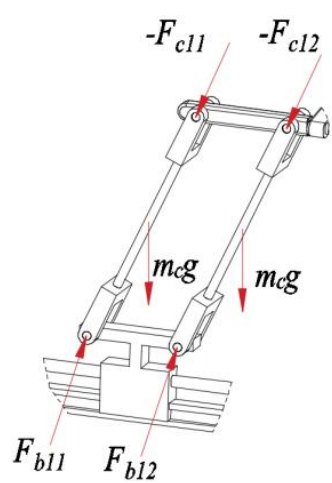

(a) $-\mathrm{P}^{(4 \mathrm{R})}$-Parallel link

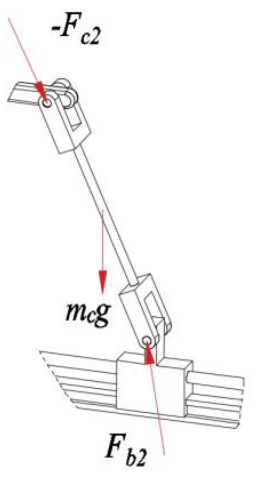

(b) Single link
Figure 6 Force analysis of -R-R-link

Therefore, the dynamic equations of the two parallel links in the parallelogram are as follows.

$$
\begin{gathered}
-\boldsymbol{F}_{c 1 i}+m_{c} g+\boldsymbol{F}_{b 1 i}=m_{c} \boldsymbol{a}_{l i},(i=1,2) \\
\frac{l_{c}}{2} \boldsymbol{c}_{i} \times\left(-\boldsymbol{F}_{c i}\right)+\frac{l_{c}}{2}\left(-\boldsymbol{c}_{i}\right) \times \boldsymbol{F}_{b i}={ }^{o} \boldsymbol{I}_{l i} \boldsymbol{\varepsilon}_{l i}+\boldsymbol{\omega}_{l i} \times\left({ }^{o} \boldsymbol{I}_{l i} \boldsymbol{\omega}_{l i}\right)
\end{gathered}
$$

Dynamic equation of single link $\left(B_{2} C_{2}\right)$ is as follows.

$$
\begin{gathered}
-\boldsymbol{F}_{c 2}+m_{c} g+\boldsymbol{F}_{b 2}=m_{c} \boldsymbol{a}_{l 2} \\
\frac{l_{c}}{2} \boldsymbol{c}_{2} \times\left(-\boldsymbol{F}_{c 2}\right)+\frac{l_{c}}{2}\left(-\boldsymbol{c}_{2}\right) \times \boldsymbol{F}_{b 2}={ }^{o} \boldsymbol{I}_{l 2} \boldsymbol{\varepsilon}_{l 2}+\boldsymbol{\omega}_{l 2} \times\left({ }^{o} \boldsymbol{I}_{l 2} \boldsymbol{\omega}_{l 2}\right)
\end{gathered}
$$

Where, ${ }^{o} \boldsymbol{I}_{l i}={ }^{o} \boldsymbol{R}_{l i} \boldsymbol{I}_{l i}{ }^{o} \boldsymbol{R}_{l i}^{T}(i=1,2),{ }^{o} \boldsymbol{I}_{l i}$ is the inertia tensor of the connecting rod in the base frame ; $\boldsymbol{I}_{l i}$ is the inertia tensor of the connecting rod in the local frame; ${ }^{o} \boldsymbol{R}_{l i}$ is the transformation matrix from the local frames of the connecting rod to the base frame.

Further, $-S$-S-link $\left(B_{3} C_{3}\right)$ is subject to the constraint reaction force of the moving platform $-\boldsymbol{F}_{a 2}$, the constraint force of the drive member $\boldsymbol{F}_{b 3}$, the self-gravity $m_{c}$ 'g, while $m_{c}$ 'is the mass of $-S-S$ - connecting rod, and its stress is shown in Figure 7.

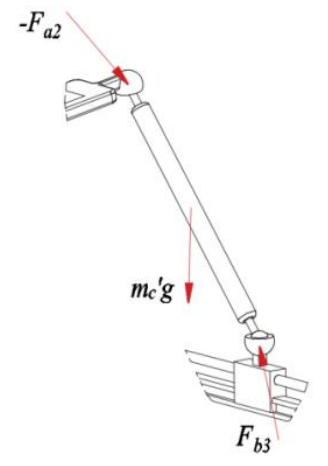

Figure 7 Force analysis diagram of -S-S- Link

Then, the dynamic equations of the -S-S- link are described as

$$
\begin{gathered}
-\boldsymbol{F}_{a 2}+m_{c}{ }^{\prime} g+\boldsymbol{F}_{b 3}=m_{c}{ }^{\prime} \boldsymbol{a}_{l 3} \\
\frac{l_{c}{ }^{\prime}}{2} \boldsymbol{c}_{3} \times\left(-\boldsymbol{F}_{a 2}\right)+\frac{l_{c}{ }^{\prime}}{2}\left(-\boldsymbol{c}_{3}\right) \times \boldsymbol{F}_{b 3}={ }^{o} \boldsymbol{I}_{l 3} \boldsymbol{\varepsilon}_{l 3}+\boldsymbol{\omega}_{l 3} \times\left({ }^{o} \boldsymbol{I}_{l 3} \boldsymbol{\omega}_{l 3}\right)
\end{gathered}
$$

where, ${ }^{o} \boldsymbol{I}_{l 3}={ }^{o} \boldsymbol{R}_{l 3} \boldsymbol{I}_{l 3}{ }^{o} \boldsymbol{R}_{l 3}^{T}$

\subsection{Dynamic equation of driving sliders}

The three driving sliders are subject to the constraint reaction forces of each link-F $b i(i=1,2,3)$, its own gravity, and the driving force of the driving motor $\mathrm{m}_{i} \mathrm{~g}(\mathrm{i}=1,2,3)$, and the force diagram of the slider is shown in Figure 8.

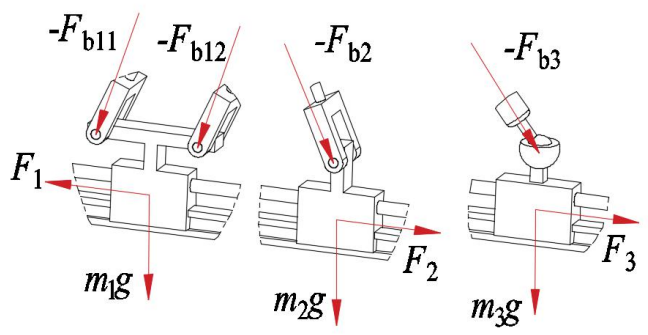

(a) Slider $1\left(A_{1}\right)$ (b) Slider $2\left(A_{2}\right)$ (c) Slider $3\left(A_{3}\right)$

Figure 8 Force analysis of three drive sliders

The dynamic equation of slider 1 is

$$
\boldsymbol{F}_{1}-\boldsymbol{F}_{b 11}-\boldsymbol{F}_{b 12}+m_{1} g=m_{1} \boldsymbol{a}_{1}
$$

The dynamic equations of slider 2 and slide 3 are as follows.

$$
\boldsymbol{F}_{i}-\boldsymbol{F}_{b i}+m_{i} g=m_{i} \boldsymbol{a}_{i},(i=2,3)
$$

\subsection{The integrated dynamic model of the PM}


The establishment of the integrated dynamic model is to eliminate the internal forces of members and to obtain the dynamic relationship between the input force, torque and output force.

Taking the dot product of the both sides of $\operatorname{Eq}(29)$ with $\boldsymbol{e}_{1}^{T}$

$$
\tau_{1}=\boldsymbol{e}_{1}^{T} \cdot \boldsymbol{F}_{1}=\boldsymbol{e}_{1}^{T} \cdot\left(\boldsymbol{F}_{b 11}+\boldsymbol{F}_{b 12}\right)+m_{1} a_{1}
$$

Where, $\tau_{i}(i=1,2,3)$ is the driving force of the slider, $\boldsymbol{e}_{i}(i=1,2,3)$ is the unit vector for driving force.

Substituting Eq. (23) into Eq .(31), we can write:

$$
\tau_{1}=\boldsymbol{e}_{1}^{T} \cdot \sum_{i=1}^{2}\left(\boldsymbol{F}_{c 1 i}-m_{c} g+m_{c} a_{l i}\right)+m_{1} a_{1}
$$

According to Eq.(24), we can write:

$$
\begin{gathered}
l_{c} \boldsymbol{c}_{1} \times\left(\boldsymbol{F}_{c_{11}}+\boldsymbol{F}_{c_{12}}\right)=\boldsymbol{C}_{1} \\
\boldsymbol{C}_{1}=l_{c} \boldsymbol{c}_{1} \times\left(m_{c} g-m_{c} \boldsymbol{a}_{l 1}\right)-2^{o} \boldsymbol{I}_{l 1} \boldsymbol{\varepsilon}_{l 1}-2 \boldsymbol{\omega}_{l 1} \times\left({ }^{o} \boldsymbol{I}_{l 1} \boldsymbol{\omega}_{l 1}\right)
\end{gathered}
$$

Taking the cross product of the two sides of Eq.(33) with $\boldsymbol{e}_{1}$ gives:

$$
l_{c} \boldsymbol{e}_{1} \times \boldsymbol{c}_{1} \times\left(\boldsymbol{F}_{c 11}+\boldsymbol{F}_{c 12}\right)=\boldsymbol{e}_{1} \times \boldsymbol{C}_{1}
$$

Then,we can write:

$$
\boldsymbol{F}_{c 11}+\boldsymbol{F}_{c 12}=\frac{\boldsymbol{c}_{1}\left[\boldsymbol{e}_{1}^{T}\left(\boldsymbol{F}_{c 11}+\boldsymbol{F}_{c 12}\right)\right]}{\boldsymbol{e}_{1}^{T} \cdot \boldsymbol{c}_{1}}-\frac{\boldsymbol{e}_{1} \times \boldsymbol{C}_{1}}{l_{c} \boldsymbol{e}_{1}^{T} \boldsymbol{c}_{1}}
$$

According to Eq.(32), we can get:

$$
\boldsymbol{e}_{1}^{T}\left(\boldsymbol{F}_{c 11}+\boldsymbol{F}_{c 12}\right)=\tau_{1}+2 \boldsymbol{e}_{1}^{T}\left(m_{c} g-m_{c} \boldsymbol{a}_{l 1}\right)-m_{1} a_{1}
$$

Substituting Eq. (37) into Eq. (36) gives:

$$
\boldsymbol{F}_{c 11}+\boldsymbol{F}_{c 12}=\frac{\boldsymbol{c}_{1}\left[\tau_{1}+2 \boldsymbol{e}_{1}^{T}\left(m_{c} \boldsymbol{g}-m_{c} \boldsymbol{a}_{l 1}\right)-m_{1} a_{1}\right]}{\boldsymbol{e}_{1}^{T} \cdot \boldsymbol{c}_{1}}-\frac{\boldsymbol{e}_{1} \times \boldsymbol{C}_{1}}{l_{c} \boldsymbol{e}_{1}^{T} \boldsymbol{c}_{1}}
$$

Similarly,

$$
\begin{aligned}
\boldsymbol{F}_{c 2} & =\frac{\boldsymbol{c}_{2}\left[\tau_{2}+\boldsymbol{e}_{2}^{T}\left(m_{c} \boldsymbol{g}-m_{c} \boldsymbol{a}_{l 2}\right)-m_{2} a_{2}\right]}{\boldsymbol{e}_{2}^{T} \cdot \boldsymbol{c}_{2}}-\frac{\boldsymbol{e}_{2} \times \boldsymbol{C}_{2}}{l_{c} \boldsymbol{e}_{2}^{T} \boldsymbol{c}_{2}} \\
\boldsymbol{F}_{a 2} & =\frac{\boldsymbol{c}_{3}\left[\tau_{3}+\boldsymbol{e}_{3}^{T}\left(m_{c}{ }^{\prime} \boldsymbol{g}-m_{c}{ }^{\prime} \boldsymbol{a}_{l 3}\right)-m_{3} a_{3}\right]}{\boldsymbol{e}_{3}^{T} \cdot \boldsymbol{c}_{3}}-\frac{\boldsymbol{e}_{3} \times \boldsymbol{C}_{3}}{l_{c} \boldsymbol{e}_{3}^{T} \boldsymbol{c}_{3}}
\end{aligned}
$$

where,

$$
\begin{aligned}
& \boldsymbol{C}_{2}=l_{c} \boldsymbol{c}_{2} \times\left(m_{c} \boldsymbol{g}-m_{c} \boldsymbol{a}_{l 2}\right)-{ }^{o} \boldsymbol{I}_{l 2} \boldsymbol{\varepsilon}_{l 2}-\boldsymbol{\omega}_{l 2} \times\left({ }^{o} \boldsymbol{I}_{l 2} \boldsymbol{\omega}_{l 2}\right) \\
& \boldsymbol{C}_{3}=l_{c}{ }^{\prime} \boldsymbol{c}_{3} \times\left(m_{c}{ }^{\prime} \boldsymbol{g}-m_{c}{ }^{\prime} \boldsymbol{a}_{l 3}\right)-{ }^{o} \boldsymbol{I}_{l 3} \boldsymbol{\varepsilon}_{l 3}-\boldsymbol{\omega}_{l 3} \times\left({ }^{o} \boldsymbol{I}_{l 3} \boldsymbol{\omega}_{l 3}\right)
\end{aligned}
$$

Substituting Eqs. (38) and (39) into Eq. (22), we can get

$$
\boldsymbol{F}_{a 1}=m_{l} g+\boldsymbol{F}_{c 11}+\boldsymbol{F}_{c 12}+\boldsymbol{F}_{c 2}-m_{l} \boldsymbol{a}_{o}
$$

Substituting Eqs. (40) and (41) into Eq. (20) and Eq. (21), we have

$$
\left[\begin{array}{c}
\boldsymbol{D} \\
\boldsymbol{E}
\end{array}\right]_{6 \times 1}=\boldsymbol{J}_{\tau} \cdot \boldsymbol{\tau}+\left[\begin{array}{c}
\boldsymbol{F}_{w} \\
\boldsymbol{M}_{w}
\end{array}\right]_{6 \times 1}
$$

where

$$
\begin{aligned}
& \boldsymbol{D}=-\frac{\boldsymbol{c}_{1}\left[2 \boldsymbol{e}_{1}^{T}\left(m_{c} \boldsymbol{g}-m_{c} \boldsymbol{a}_{l 1}\right)-m_{1} a_{1}\right]}{\boldsymbol{e}_{1}^{T} \boldsymbol{c}_{1}}-\frac{\boldsymbol{c}_{2}\left[\boldsymbol{e}_{2}^{T}\left(m_{c} \boldsymbol{g}-m_{c} \boldsymbol{a}_{l 2}\right)-m_{2} a_{2}\right]}{\boldsymbol{e}_{2}^{T} \boldsymbol{c}_{2}} \\
& +\sum_{i=1}^{2} \frac{\boldsymbol{e}_{i} \times \boldsymbol{C}_{i}}{l_{c} \boldsymbol{e}_{i}^{T} \boldsymbol{c}_{i}}-\frac{\boldsymbol{c}_{3}\left[\boldsymbol{e}_{3}^{T}\left(m_{c}^{\prime} \boldsymbol{g}-m_{c}{ }^{\prime} \boldsymbol{a}_{l 3}\right)-m_{3} a_{3}\right]}{\boldsymbol{e}_{3}^{T} \boldsymbol{c}_{3}}+\frac{\boldsymbol{e}_{3} \times \boldsymbol{C}_{3}}{l_{c} \boldsymbol{e}_{3}^{T} \boldsymbol{c}_{3}}-m_{l}\left(\boldsymbol{g}-\boldsymbol{a}_{o}\right) \\
& -m\left(\boldsymbol{g}-\boldsymbol{a}_{p}\right) \\
& \boldsymbol{E}=\frac{1}{2} \boldsymbol{c}_{p} \times\left[\frac{\boldsymbol{c}_{1}\left[2 \boldsymbol{e}_{1}^{T}\left(m_{c} \boldsymbol{g}-m_{c} \boldsymbol{a}_{l 1}\right)-m_{1} a_{1}\right]}{\boldsymbol{e}_{1}^{T} \boldsymbol{c}_{1}}+\frac{\boldsymbol{c}_{2}\left[\boldsymbol{e}_{2}^{T}\left(m_{c} \boldsymbol{g}-m_{c} \boldsymbol{a}_{l 2}\right)-m_{2} a_{2}\right]}{\boldsymbol{e}_{2}^{T} \boldsymbol{c}_{2}}\right. \\
& \left.\sum_{i=1}^{2} \frac{\boldsymbol{e}_{i} \times \boldsymbol{C}_{i}}{l_{c} \boldsymbol{e}_{i}^{T} \boldsymbol{c}_{i}}+m_{l}\left(\boldsymbol{g}-\boldsymbol{a}_{o}\right)\right]-\frac{1}{2} \boldsymbol{c}_{p} \times\left[\frac{\boldsymbol{c}_{3}\left[\boldsymbol{e}_{3}^{T}\left(m_{c}{ }^{\prime} \boldsymbol{g}-m_{c}{ }^{\prime} \boldsymbol{a}_{l 3}\right)-m_{3} a_{3}\right]}{\boldsymbol{e}_{3}^{T} \boldsymbol{c}_{3}}-\frac{\boldsymbol{e}_{3} \times \boldsymbol{C}_{3}}{l_{c} \boldsymbol{e}_{3}^{T} \boldsymbol{c}_{3}}\right]- \\
& { }^{o} \boldsymbol{I}_{p} \boldsymbol{\varepsilon}_{p}-\boldsymbol{\omega}_{p} \times\left({ }^{o} \boldsymbol{I}_{p} \boldsymbol{\omega}_{p}\right)
\end{aligned}
$$$$
\boldsymbol{J}_{\tau}=\left[\begin{array}{ccc}
\frac{\boldsymbol{c}_{1}}{\boldsymbol{e}_{1}^{T} \boldsymbol{c}_{1}} & \frac{\boldsymbol{c}_{2}}{\boldsymbol{e}_{2}^{T} \boldsymbol{c}_{2}} & \frac{\boldsymbol{c}_{3}}{\boldsymbol{e}_{3}^{T} \boldsymbol{c}_{3}} \\
-\frac{\boldsymbol{c}_{p} \times \boldsymbol{c}_{1}}{2 \boldsymbol{e}_{1}^{T} \boldsymbol{c}_{1}} & -\frac{\boldsymbol{c}_{p} \times \boldsymbol{c}_{2}}{2 \boldsymbol{e}_{2}^{T} \boldsymbol{c}_{2}} & \frac{\boldsymbol{c}_{p} \times \boldsymbol{c}_{3}}{2 \boldsymbol{e}_{3}^{T} \boldsymbol{c}_{3}}
\end{array}\right]_{6 \times 3}
$$$$
\boldsymbol{\tau}=\left[\begin{array}{lll}
\tau_{1} & \tau_{2} & \tau_{3}
\end{array}\right]^{T}{ }_{3 \times 1}
$$

According to Eq. (42), we can get:

$$
\boldsymbol{\tau}=\boldsymbol{J}_{\tau}^{-1} \cdot\left[\begin{array}{c}
\boldsymbol{D} \\
\boldsymbol{E}
\end{array}\right]-\boldsymbol{J}_{\tau}^{-1} \cdot\left[\begin{array}{c}
\boldsymbol{F}_{w} \\
\boldsymbol{M}_{w}
\end{array}\right]
$$

When the motion law of the moving platform and the external force and torque are known, the driving force of each driving pair can be obtained from Eq.(43).

\section{Dynamic Simulation}

Firstly, the following motion laws of three driving pairs are given.

$$
\left\{\begin{array}{l}
l_{1}=10 \cos (t)-27 \\
l_{2}=-10 \cos (t)+27 \\
l_{3}=-10 \cos (t)+14
\end{array}\right.
$$

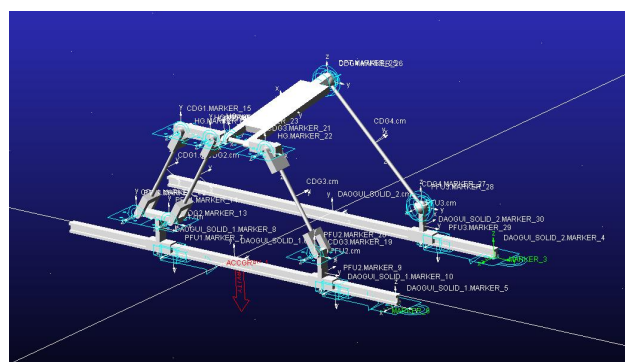


Figure 9 Virtual prototype of 2T1R parallel mechanism

A three-dimensional prototype of the PM is designed, as shown in Figure 9. The dimension parameters of the PM are shown in Table 1.

Table 1 Dimension Parameters of the 2T1R PM

\begin{tabular}{cc}
\hline Size parameters & Value \\
\hline$l_{b} / \mathrm{mm}$ & 10 \\
$l_{d} / \mathrm{mm}$ & 17 \\
$l_{c} / \mathrm{mm}$ & 43.8 \\
$l_{c} / \mathrm{mm}$ & 60 \\
$-\mathrm{XA}_{3} / \mathrm{mm}$ & 60 \\
$2 l_{p} / \mathrm{mm}$ & 54 \\
$m_{1} / \mathrm{kg}$ & 0.00254 \\
$m_{2} / \mathrm{kg}$ & 0.00162 \\
$m_{3} / \mathrm{kg}$ & 0.00153 \\
$m_{c} / \mathrm{kg}$ & 0.00285 \\
$m_{c} / \mathrm{kg}$ & 0.00129 \\
$m_{l} / \mathrm{kg}$ & 0.00491 \\
$m / \mathrm{kg}$ & 0.0131 \\
\hline
\end{tabular}

The above parameters are substituted into the dynamic Eqs. $(20) \sim(43)$, and the driving forces of the three driving pairs are calculated by MATLAB. The curves of the driving forces are shown in Figure 10 (a).

Then, the three-dimensional prototype is input into ADAMS, and the material properties of each part and the constraint types of the kinematic pairs are specified. The vertical downward gravity is applied, and the simulation step of $0.01 \mathrm{~s}$ and the simulation time of $10 \mathrm{~s}$ are selected for dynamic simulation of the virtual prototype.

As shown in Fig.10 (b), the calculated value is compared with the simulation result of ADAMS, which shows that the simulation value is basically consistent with the theoretical value. The maximum error of the driving force of each pair is $1.32 \%, 5.8 \%$ and $5.2 \%$, which verifies the correctness of the dynamic model.

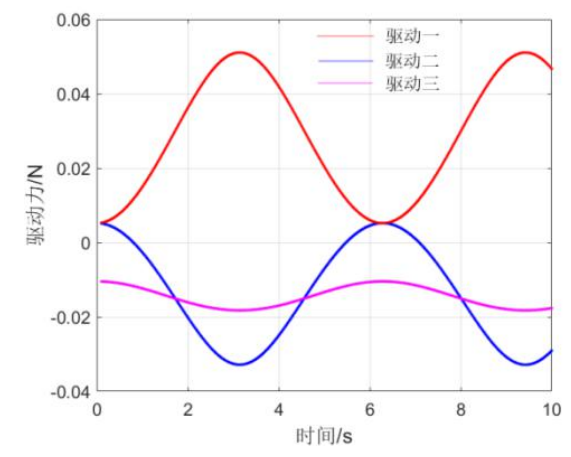

(a) Theoretical driving force

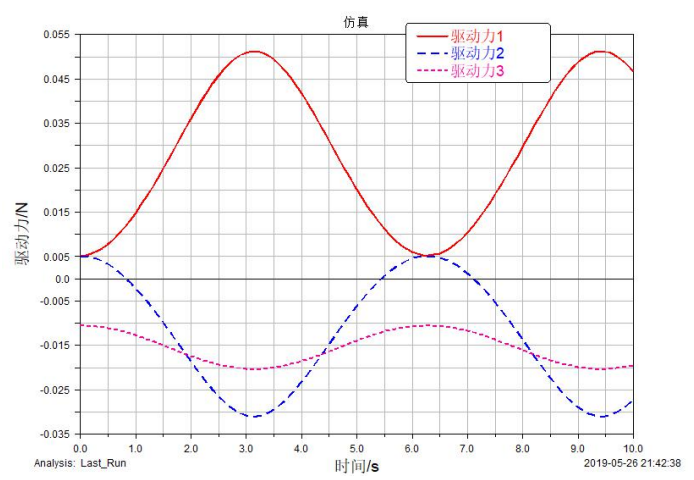

(b) Driving force simulation value

Figure 10 Driving Force of Each Driving Pair

Under the same simulation duration, different steps are selected to record the calculation time of MATLAB, as shown in Table 2.

Table 2 Matlab calculation time

\begin{tabular}{ccc}
\hline $\begin{array}{c}\text { Simulation } \\
\text { duration / s }\end{array}$ & Step size / s & $\begin{array}{c}\text { Calculation } \\
\text { time / s }\end{array}$ \\
\hline 10 & 0.01 & 4.77 \\
10 & 0.005 & 9.13 \\
10 & 0.004 & 11.64 \\
\hline
\end{tabular}

As shown in Table 2, only when the step size $\leqslant$ $\frac{10}{0.005}=2000$, the dynamic modeling analysis program is real-time .

\section{Conclusions}

For a new type of 3-DOF PM proposed in this paper, the analytical solution of forward kinematics is given, and it is used to analyze the velocity and acceleration. The driving force is obtained by calculating the dynamic equation. The dynamic simulation of the three-dimensional prototype is 
carried out by ADAMS, and the simulation value of driving force is obtained. By comparing the two cases, the correctness of the dynamic modeling is verified, which lays a foundation for the design, manufacture and application of PM.

\section{Declaration}

\section{Acknowledgement}

The first authors sincerely thanks to Professor Shen of Changzhou University for his critical discussion and reading during manuscript preparation.

\section{Funding}

Supported by National Natural Science Foundation of China (Grant No.51975062) and National Science Foundation of Jiangsu Province (Grant No.BK20161192)

\section{Availability of data and materials}

The datasets supporting the conclusions of this article are included within the article.

\section{Authors' contributions}

The author' contributions are as follows: Huiping Shen was in charge of the whole work; Ke Wang wrote the manuscript.

\section{Competing interests}

The authors declare no competing financial interests.

\section{Consent for publication \\ Yes}

\section{Ethics approval and consent to participate}

Yes

\section{References}

[1] H P Shen, M Lv, X R Zhu, Y F Li. Topological design and kinematics of a single-degree-of-freedom 3T1R parallel mechanism [J] .China Mechanical Engineering, 2019, 30 (08): 961-968.

[2] T L Yang, A X Liu, H P Shen, et al. Topological Design of Robot Mechanism. Beijing: Science Press, 2012.05.

[3] B Y Chang, X N Li, G G Jin, Z Zhang, S Yang. Kinematics analysis of a 3T1R parallel mechanism with full-circle rotation capability [J]. Journal of Agricultural Machinery,2019, 50(7): 406-416.

[4] Moussab Bennehar, Ahmed Chemori, François Pierrot. A new revised desired compensation adaptive control for enhanced tracking: application to RA-PKMs[J]. Advanced Robotics, 2016, 30(17-18): 1192-1214.

[5] H P Shen, K Xu, T L Yang, J M Deng. Design and Kinematics of a New 3T1R Parallel Manipulator 2- (RPa3R) 3R with Zero Coupling and Motion Decoupling [J] .Journal of Mechanical Engineering, 2019, 55 (05): 53-64.
[6] W Liu, H Z Liu. Synthesis of Parallel Mechanisms with 2T1Rand 3R Motion Modes [J] .Chinese Journal of Mechanical Engineering, 2019, 55 (03): 53-63.

[7] BHASKAR D, MRUTHYUNJAYA T S. A Newton-Euler formulation for the inverse dynamics of the Stewart platform manipulator[J]. Mechanism and Machine Theory, 1998, 33 (8): 1135-1152.

[8] Naser M, Alireza A, Jaspreet D, et al. A comprehensive inverse dynamics problem of a stewart platform by means of Lagrangian formulation[A]. ASME 2017 Dynamic Systems and Control Conference. Virginia: DSCC 2017-5098.

[9] Y F Zhang, P Jin, J L Gong, Q Liu. Dynamic modeling of viscous friction conditions of 3-RPS parallel robot [J]. Transactions of the Chinese Society of Agricultural Machinery, 2018,49 (09): 374- 381.

[10] Y B Li, H Zheng, P Sun. Dynamic Modeling and Coupling Analysis of 5-PSS/UPU Parallel Mechanism Considering Joint Friction [J]. Chinese Journal of Mechanical Engineering, 2019,55 (03) : 43-52.

[11] Fu S, Yao Y, Wu Y. Comments on "A Newton-Euler formulation for the inverse dynamics of the Stewart platform manipulator"'[J]. Mechanism and Machine Theory, 2007, 42, 1668-1671.

[12] Pedrammehr S, Mahboubkhah M, Khani, N. Improved dynamic equations for the generally configured Stewart platform manipulator[J] Mechanical Science and Technology. 2012, 26(3), 711-721.

[13] X L Chen, W M Feng, Y S Zhao. Dynamic Model of a 5-DOF Parallel Robot Mechanism[J].Transactions of the Chinese Society of Agricultural Machinery, 2013,44 (01): 236-243.

[14] M C Geng, T S Zhao, C Wang, et al. Dynamic analysis of 4-UPS/UPR parallel mechanism[J]. Transactions of the Chinese Society of Agricultural Machinery, 2014, 45 (8): 299-306.

[15] Y G Li, Y M Song, Z Y Feng, C Zhang. Inverse dynamics analysis of 3-RPS parallel mechanism based on Newton's Euler method [J] .Acta Aeronautica, 2007 (05): 1210-1215.

[16] S Z Liu, Y Q Yu, G N Tong, J X Yang. Kinematics and Dynamics Analysis of a 3 Degrees of Freedom Parallel Robot [J] .Chinese Journal of Mechanical Engineering, 2009, 45 (08): 11- 17

[17] H P Shen, J M Deng, K Wang. A two translation and one rotation parallel mechanism with zero coupling and positive solution of analytical position, China, 201961623865x, 2019.6.11

\section{Biographical notes}

Ke Wang born in 1994, is currently a engineer at Black \& Decker(Suzhou) Technology Limited Company, China. He received his master degree from Changzhou University, China, in 2020. E-mail: wangk1994@163.com

Ju Li, born in 1981, is currently a Vice Professor at Changzhou University, China. Her main research interests include parallel mechanism, kinematics, E-mail: wangju0209@163.com 
Huiping Shen * born in 1965, is currently a professor and a $\mathrm{PhD}$ candidate supervisor at Changzhou University, China. His main research interests include parallel mechanism, kinematics, institutional topology.

E-mail: Shp65@126.com

Ting-Li Yang, born in 1940, is currently a guest professor at Changzhou University, China. His main research interest include modern mechanisms, basic theory of robotic mechanism.
Jingjing You, born in 1985, is currently a professor and a Master supervisor at College of Mechanical and Electronic Engineering, Nanjing Forestry University, China.

E-mail: youjingjing251010@126.com

E-mail:yangti@126.com 
Figures

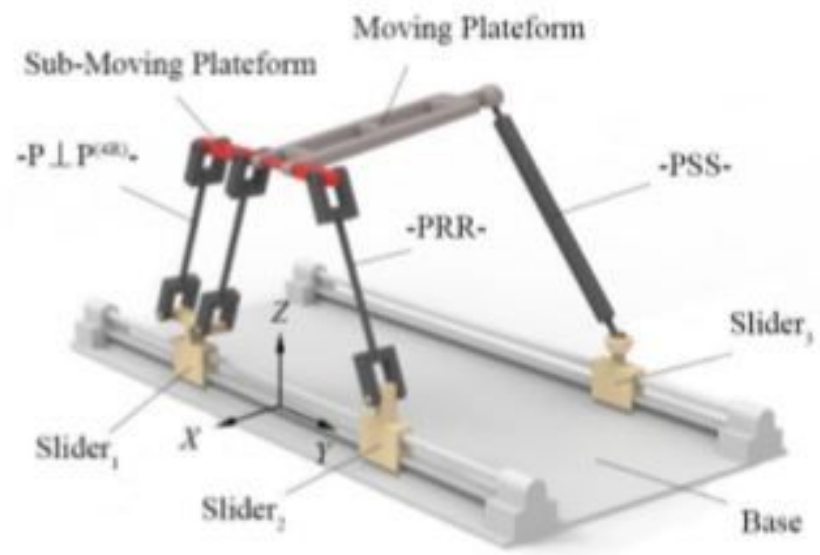

Figure 1

3D model of a $2 T 1 R$ PM

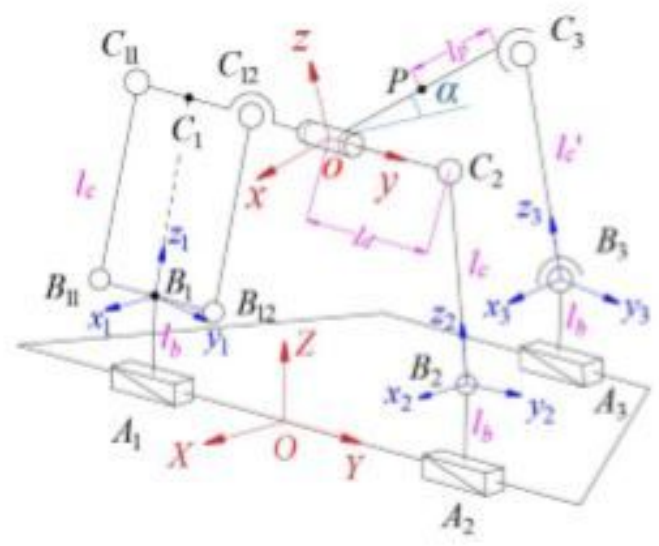

Figure 2

Schematic diagram of 2T1R PM

$$
{ }^{o} \boldsymbol{R}_{h_{3}}=\boldsymbol{R}\left(Y, \theta_{1}\right) \boldsymbol{R}\left(x_{3}^{\prime}, \theta_{2}\right)
$$

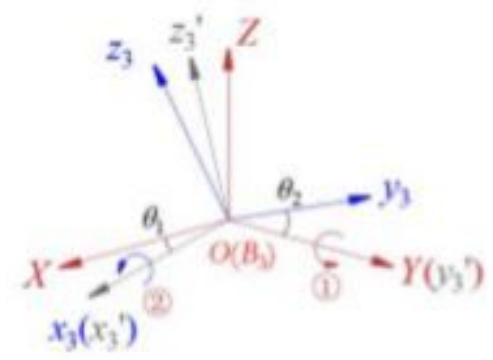

Figure 3 
Coordinate system B3-x3y3z3 z Euler transform

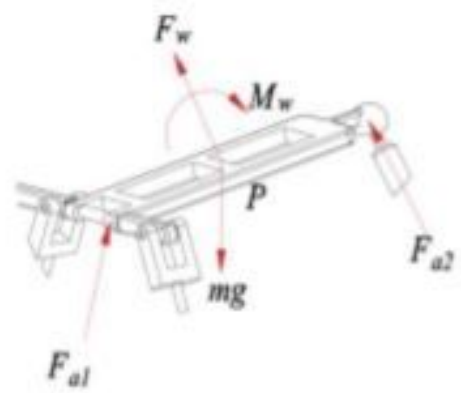

Figure 4

Force analysis of the moving platform

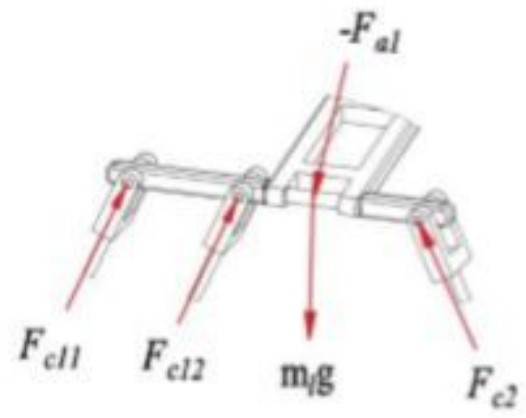

\section{Figure 5}

Force analysis of sub-moving platform

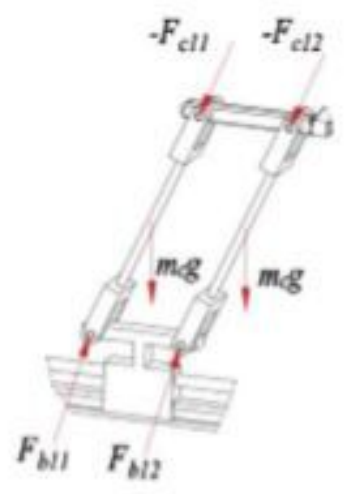

(a) $-\mathrm{P}^{(4 \mathrm{R})}-\mathrm{Parallel}$ link

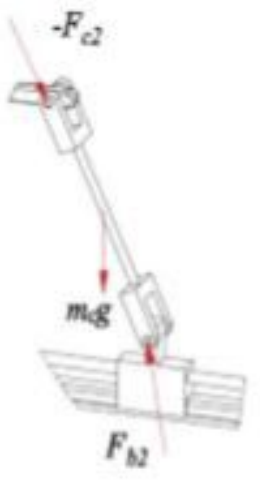

(b) Single link

Figure 6

Force analysis of-R-R-link 


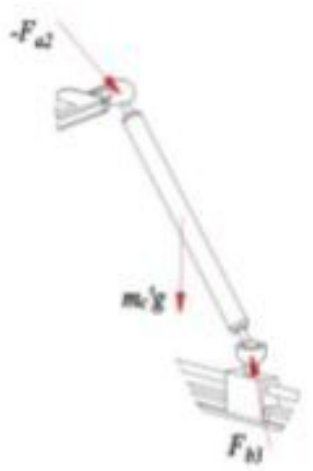

\section{Figure 7}

Force analysis diagram of-S-S-Link

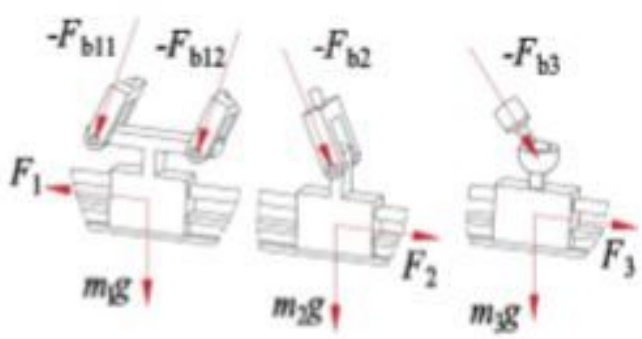

\section{Figure 8}

Force analysis of three drive sliders

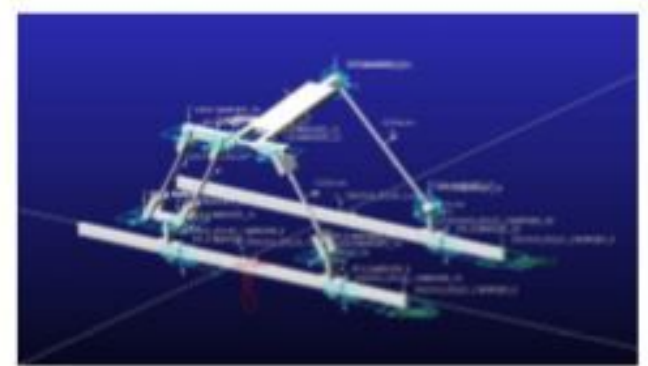

Figure 9

Virtual prototype of $2 \mathrm{~T} 1 \mathrm{R}$ parallel mechanism 


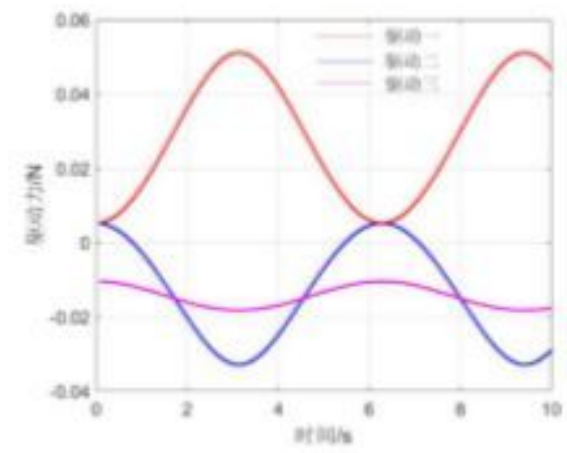

(a) Theoretical driving force

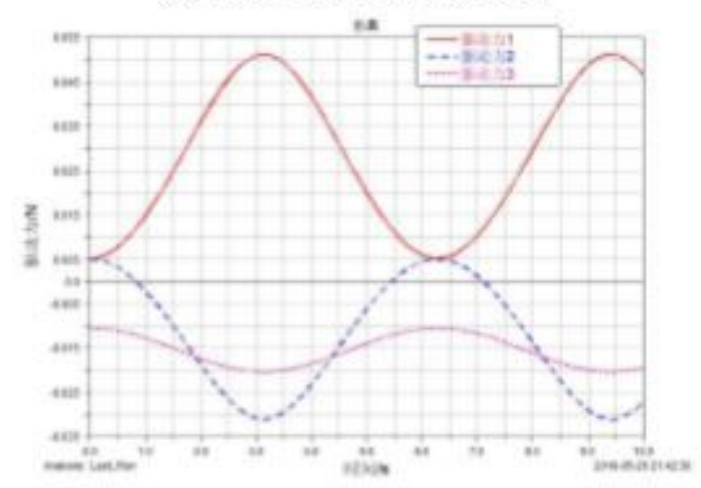

(b) Driving force simulation value

\section{Figure 10}

Driving Force of Each Driving Pair 